\title{
Clinical \& Surgical Outcomes of Shoulder Arthrodesis: A Cohort of Seven Cases from the South Metropolitan Health Service of Western Australia
}

\author{
Kieran Bochat, MBBS, Sheldon M Moniz, MBBS ${ }^{*}$ and Andrew C Mattin, MBBS, FRACS
}

Department of Orthopaedic Surgery, Fremantle Hospital, Australia

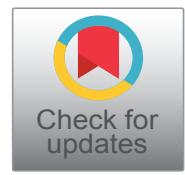

*Corresponding author: Dr. Sheldon M Moniz, Department of Orthopaedic Surgery, Fremantle Hospital, Alma St, Fremantle WA 6160, Australia

\begin{abstract}
Background: Shoulder arthrodesis is an uncommon surgical procedure performed for a range of shoulder pathology including brachial plexus injury, instability or as a salvage procedure for failed arthroplasty and tumour cases. Cohorts report generally good outcomes despite high rates of complications. We aim to investigate the outcomes of all patients undergoing shoulder arthrodesis within the South Metropolitan Health Service (SMHS) from 2001-2019. There is a gap in the literature about the social demographic of patients undergoing shoulder arthrodesis. We expect a high incidence of confounding factors including multiple previous surgeries, unemployment, cigarette smoking and drug abuse to impact patient outcomes.

Method: A comprehensive search of the SMHS electronic theatre databases was performed, spanning years 20012019. Patients undergoing shoulder arthrodesis were identified. Clinical records and imaging were then reviewed to determine patient demographics and outcomes.

Results: Shoulder arthrodesis is an extremely uncommon procedure, with only seven recorded cases over 19 years. $71 \%$ of patients suffered from drug addiction, and $57 \%$ were smokers. Rate of shoulder fusion was $71 \%$ at a mean of 17 months. All employed patients returned to work including one as a manual labourer. $43 \%$ had ongoing pain, all of which were chronic pain sufferers with pre-existing opioid addiction. $29 \%$ of patients required further surgery for infection and persistent pain. Patients being treated for instability were frustrated by reduced range of motion post-operatively.

Conclusion: Shoulder arthrodesis is an uncommon procedure with reliable outcomes despite high rates of complications. Drug abuse, cigarette smoking and unemployment rates are high within our cohort. It has utility for employed patients including manual labourers. Pre-operative counselling is of particular importance in patients undergoing shoulder arthrodesis for instability symptoms.
\end{abstract}

Level of Evidence: IV

\section{Keywords}

Shoulder arthrodesis, Glenohumeral joint arthrodesis, Social factors, Patient outcomes, Surgical outcomes

\section{Introduction}

Shoulder arthrodesis is an uncommon surgical procedure. A sparse number of retrospective cohorts appear in the literature. It is performed for a wide range of clinical presentations including brachial plexus injury (traumatic or iatrogenic), recurrent shoulder instability, failed shoulder arthroplasty, and tumour cases. Patients have often had multiple previous surgeries, with arthrodesis employed as a salvage procedure [1,2]. Most cohorts report good outcomes for patients at long term follow-up despite a high incidence of complications and re-operation rates. We aim to add to the body of evidence, specifically patient demographics and social factors. We anticipate our cohort to have a high incidence of confounding social factors that increase the risk for high-energy trauma, late presentation and non-compliance.

\section{Indications}

In the neurologically compromised patient, shoulder arthrodesis allows the scapulothoracic joint to transfer meaningful movement to the elbow, wrist and hand. Vander Lingen, et al. demonstrated improvements in pain, function and strength for brachial plexus injury patients post-shoulder arthrodesis [3]. In another cohort

Citation: Bochat K, Moniz SM, Mattin AC (2020) Clinical \& Surgical Outcomes of Shoulder Arthrodesis: A Cohort of Seven Cases from the South Metropolitan Health Service of Western Australia. Int Arch Orthop Surg 3:019. doi.org/10.23937/2643-4016/1710019

Accepted: June 27, 2020; Published: June 29, 2020

Copyright: (c) 2020 Bochat K, et al. This is an open-access article distributed under the terms of the Creative Commons Attribution License, which permits unrestricted use, distribution, and reproduction in any medium, provided the original author and source are credited. 
of eight patients with obstetric brachial plexus injuries all were satisfied with the outcome of shoulder arthrodesis with improvements in their range of motion [4].

Thangarajah, et al. evaluated a cohort of eight patients who underwent shoulder arthrodesis for pain and dysfunction secondary to recurrent instability [1]. Instability scores and subjective shoulder value improved. Scalise and lannotti analyzed seven patients who had failed shoulder arthroplasty and went on to arthrodesis. At an average of 4 years follow-up (range 1.5-8 years) subjective shoulder function and pain were significantly improved $(p=0.008)[5]$.

\section{Surgical considerations}

Rowe described the goals of shoulder arthrodesis in 1974 [6]. First, the hand should reach the face, head and body midline both anteriorly and posteriorly. Second, the arm should be in a position of maximum strength to lift, push and pull. Third, the shoulder should be comfortable when the arm is at rest against the trunk, without scapula winging [6]. The optimal position of the fused arm remains unclear. The majority of literature supports $15^{\circ}$ to $25^{\circ}$ of forward flexion and abduction and $40^{\circ}$ to $45^{\circ}$ of internal rotation [6-9]. Souza, et al. reported that excessive abduction and external rotation lead to winging of scapula at rest, which results in pain [10]. Wagner, et al. observed that abduction or flexion of $\geq 25^{\circ}$ led to better shoulder and extremity function [7]. Van der Lingen, et al. and Chammas, et al. found malposition of the humerus leads to poorer outcomes [3].

The use of plate fixation and sufficient autograft is well supported in the literature $[5,8,9,11-13]$. Screw fixation was associated with a lower rate of postoperative fractures and implant removal for irritation in a cohort of 43 patients, but the use of plates and bone-grafting improved the rates of union [12]. Atlan, et al. found that cortico-cancellous autograft in the subacromial space significantly increased fusion ( $43 \%$ vs. $4 \% \mathrm{P}<0.001$ ) in their cohort of 54 patients [11]. Wagner, et al. also found the inclusion of the acromion in the fusion was crucial to prevent non-union [7].

\section{Complications}

Most cohorts report a high incidence of complications and return to theatre. Miller, et al. reviewed outcomes of 11 children suffering polio who underwent shoulder arthrodesis for flail shoulder [14]. At a mean follow-up of 41 months, one patient had required revision surgery for non-union, two required corrective osteotomies, and six patients had metalwork removed due to discomfort. Chammas, et al. followed 27 patients for a mean of six years. Two (7\%) had non-unions requiring re-operation and bone graft. Three $(11 \%)$ sustained humerus fractures [15]. Wagner, et al. evaluated a diverse cohort of 29 patients at a mean follow up of 12 years (range $2-22$ years) [7]. 12 patients (41\%) had postoperative complications. These included six periprosthetic fractures, seven non-unions, and three infections. 11 patients (38\%) underwent further surgery for non-union (seven patients), debridement and lavage for deep infection (two), fracture fixation (two), and removal of metal due to irritation (one). Atlan, et al. had 10 of 54 patients (18\%) require re-operation for delayed union at a mean follow-up of 37 months [11]. Three (6\%) had not united at last follow-up. Scalise and lannotti's cohort had four of seven patients (57\%) require revision surgery with bone graft. Only two of these united while the remaining two patients (29\%) had failed to achieve union at final follow-up [5].

\section{Outcomes}

Despite high complication rates, cohorts generally have good outcomes. All patients in Miller's cohort were satisfied at the final follow-up with minimal pain [14]. In Chammas cohort, 26 of 27 had improved satisfaction and all had gone on to union [15]. Wagner, et al. demonstrated reasonable pain relief and improved shoulder stability in their cohort [7]. Atlan reports a $94 \%$ fusion rate [11]. Subjective shoulder function and pain were significantly improved in Scalise and lannotti cohort at an average of 4 years follow-up [5].

Patients having shoulder arthrodesis for recurrent instability tend to have worse outcomes than those having arthrodesis for other indications [7]. Thangarajah, et al. looked at a cohort of six young epileptic patients who had recurrent instability attributed to seizures [2]. These patients had previously had up to 11 failed procedures each (mean of four). While their instability scores improved, two of the six patients had a decreased mean subjective shoulder score. This was attributable to a loss of range of motion [2].

Shoulder arthrodesis is a viable surgical treatment for a wide range of shoulder pathology. The optimal position of the humerus is still debated. Plate and screw fixation with sufficient bone graft is the favoured surgical technique. Although complication rates are high, patients generally have good outcomes. Pre-operative counselling is important in patients with recurrent instability who need to accept a loss of range of motion to gain stability.

\section{Materials and Methods}

A comprehensive search of the electronic theatre databases for the South Metropolitan Health Service of Western Australia was performed for 2001-2019. Patients undergoing shoulder arthrodesis were identified. Clinical records and imaging were then reviewed to determine patient outcomes.

Seven shoulder arthrodesis procedures were performed. Indications for surgery were; post-traumatic osteonecrosis of the humeral head with and without brachial plexus injury, osteoarthritis with obstetric bra- 
chial plexus injury, post-traumatic osteoarthritis with and without axillary nerve palsy, post-traumatic osteoarthritis with instability, and recurrent instability due to refractory seizures. Procedures were performed by five different Consultant Orthopaedic surgeons, all with a special interest in shoulder surgery. Surgeons assessed the patient's passive ability to get the hand to mouth and face intra-operatively. Plate and screw constructs were used in all seven patients.

\section{Results}

\section{Patient characteristics}

Mean patient age was 34 (range 22-45). The majority of patients were male (five). Median American Society of Anaesthesiologists (ASA) score was 2 (range 1-3). Five patients (71\%) suffered from drug addiction (four opioid, one methamphetamine). Four (57\%) were active cigarette smokers. Five (71\%) were unemployed. Four patients (57\%) lived outside the Perth metropolitan region. One patient had 11 previous surgeries on the affected shoulder, another three procedures, while three patients had not had any previous surgery. Surgical history of the remaining two patients was unknown (Table 1).

\section{Outcomes}

Five patients (71\%) had achieved union at last follow-up. Mean time to fusion was 16 months (range 8-31 months). One did not attend follow-up beyond five months and was pain-free at last visit. The final patient had recently undergone revision shoulder arthrodesis. Both of the employed patients returned to their pre-operative occupation, including one manual labourer.

Four patients (57\%) were pain-free at last follow-up. Three (43\%) had ongoing pain beyond 12 months. All of these three patients had a pre-operative opioid addiction and were chronic pain patients. Both patients treated for instability symptoms reported frustration at their reduced range of motion postoperatively.

\section{Complications}

Two patients (29\%) required further surgery. One patient developed a deep infection and periprosthetic fracture 6 weeks post-fusion. This patient underwent two further operations; open lavage then subsequent removal of metal. The fracture was managed conservatively. The patient last attended follow-up five months after index procedure and at this time reported no pain. The second patient had loosening at the 13-month mark and underwent removal of metal and biopsy. Microbiology was negative for infection and the patient underwent revision arthrodesis.

\section{Discussion}

Shoulder arthrodesis was extremely uncommon in SMHS, with only seven recorded cases over a 19 year period. We believe high rates of drug addiction (71\%),

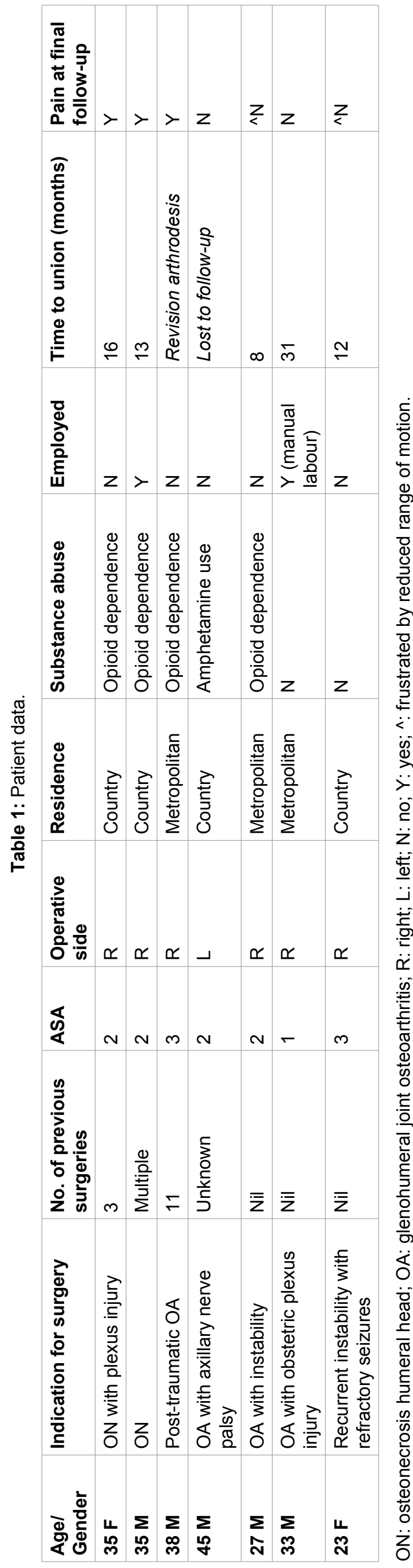


smoking (57\%) and unemployment (71\%) in our cohort contributed to high rates of chronic pain and complications. $43 \%$ of patients had ongoing pain post arthrodesis, all of which were chronic pain sufferers with pre-existing opioid addiction. $29 \%$ of patients' required further surgery for infection and non-union. Numerous other cohorts in the literature report similarly high rates of complications $[5,7,11,14,15]$.

Rates of previous surgery were lower than expected (29-57\%). The majority of our cohort lived outside the metropolitan region, meaning poorer access to health services and thus more likely to present late.

Surgical outcomes were reasonable with the rate of shoulder fusion $71 \%$ at a mean of 17 months. One patient was lost to follow-up. Shoulder arthrodesis is a viable option in employed patients including manual labourers, as all employed patients returned to work, including one as a manual labourer.

Patients having shoulder arthrodesis for instability were frustrated by reduced range of motion post-operatively, consistent with previous findings in the literature [3]. Pre-operative counselling is important in patients with recurrent instability who need to accept a loss of range of motion to gain stability.

The major limitation of our study was the small sample size. It was retrospective in nature. There was also a diverse range of indications for glenohumeral arthrodesis as opposed to a group of patients with a single pathology. Follow-up was variable in duration with a $14 \%$ (one patient) loss to follow-up.

\section{Conclusion}

Shoulder arthrodesis is an uncommon procedure with reliable outcomes despite high rates of complications. Drug abuse, cigarette smoking and unemployment rates are high within our cohort. It has utility for employed patients including manual labourers. Pre-operative counselling is of particular importance in patients undergoing shoulder arthrodesis for instability symptoms.

\section{Acknowledgements}

Richard Madayag, Clinical applications reporter, Theatre Management System, South Metropolitan Health Service of Western Australia.

\section{References}

1. Thangarajah T, Higgs D, Bayley JL, Lambert SM (2017) Glenohumeral arthrodesis for recurrent types II and III shoulder instability. J Shoulder Elbow Surg 26: 687-691.

2. Thangarajah T, Alexander S, Bayley I, Lambert SM (2014) Glenohumeral arthrodesis for the treatment of recurrent shoulder instability in epileptic patients. Bone Joint $\mathrm{J}$ 96: 1525-1529.

3. Van der Lingen MAJ, de Joode SGCJ, Schtanus MGM, Grimm B, van Nie FA, et al. (2018) Satisfied patients after shoulder arthrodesis for brachial plexus lesions even after 20 years of follow-up. Eur $\mathrm{J}$ Orthop Surg Traumatol 28: 1089-1094.

4. Bekhelyar Z, Djeffel AB, Cambon-Binder A (2019) Glenohumeral fusion in adults with sequelae of obstetrical brachial plexus injury: A report of eight cases. J Hand Surg Eur 44: 248-255.

5. Scalise JJ, lannotti JP (2008) Glenohumeral arthrodesis after failed prosthetic shoulder arthroplasty. J Bone Joint Surg Am 90: 70-77.

6. Rowe CR (1974) Re-evaluation of the position of the arm in arthrodesis of the shoulder in the adult. J Bone Joint Surg Am 56: 913-922.

7. Wagner ER, McLaughlin R, Sarfani S, Cofield RH, Sperling JW, et al. (2018) Long-term outcomes of glenohumeral arthrodesis. J Bone Joint Surg Am 100: 598-604.

8. Esenyel CZ, Ozturk K, Imren Y, Ayanoglu S (2011) Shoulder arthrodesis with plate fixation. Acta Orthop Traumatol Turc 45: 412-420.

9. Stark DM, Bennett JB, Tullos HS (1991) Rigid internal fixation for shoulder arthrodesis. Orthopedics 14: 849-855.

10. Sousa R, Pereira A, Massada M, Trigueiros M, Lemos R, et al. (2011) Shoulder arthrodesis in adult brachial plexus injury: What is the optimal position? J Hand Surg Eur 36: 541-547.

11. Atlan F, Durand S, Fox M, Levy P, Belkheyar Z, et al. (2012) Functional outcome of glenohumeral fusion in brachial plexus palsy: A report of 54 cases. J Hand Surg Am 37: 683-688.

12. Ruhmann O, Schmolke S, Bohnsack M, Flamme C, Wirth CJ (2005) Shoulder arthrodesis: Indications, technique, results, and complications. J Shoulder Elbow Surg 14: 38-50.

13. Richards RR, Beaton D, Hudson AR (1993) Shoulder arthrodesis with plate fixation: Functional outcome analysis. $J$ Shoulder Elbow Surg 2: 225-239.

14. Miller JD, Pinero JR, Goldstein R, Yen YM, Eves W, et al. (2011) Shoulder arthrodesis for treatment of flail shoulder in children with polio. J Pediatr Orthop 31: 679-682.

15. Chammas M, Goubier JN, Coulet B, Reckendorf GMZ, Picot MC, et al. (2004) Glenohumeral arthrodesis in upper and total brachial plexus palsy. $\mathrm{J}$ Bone Joint Surg $\mathrm{Br} 86$ : 692-695. 